\title{
Positions et stratégies des éditeurs dans le champ éditorial québécois
}

\section{The positions and strategies of Quebec publishers \\ Las posiciones y estrategias de los editores en el mundo editorial quebecense}

\section{Ignace $\mathrm{Cau}$}

Volume 26, numéro 3, septembre 1980

URI : https://id.erudit.org/iderudit/1054227ar

DOI : https://doi.org/10.7202/1054227ar

Aller au sommaire du numéro

Éditeur(s)

Association pour l'avancement des sciences et des techniques de la documentation (ASTED)

ISSN

0315-2340 (imprimé)

2291-8949 (numérique)

Découvrir la revue

Citer cet article

Cau, I. (1980). Positions et stratégies des éditeurs dans le champ éditorial québécois. Documentation et bibliothèques, 26(3), 139-149.

https://doi.org/10.7202/1054227ar

\section{Résumé de l'article}

Après avoir identifié quatre axes possibles, l'auteur analyse le champ éditorial de différentes maisons québécoises de 1960 à 1977 et dégage l'image de ces éditeurs par rapport au marché et à leur stratégie éditoriale. L'évolution de l'édition devient laïque et idéologique au cours de cette période, mais est toujours faiblement structurée et partagée entre le livre " culturel » et le livre "pratique ".
Tous droits réservés ( $)$ Association pour l'avancement des sciences et des techniques de la documentation (ASTED), 1980
Ce document est protégé par la loi sur le droit d'auteur. L'utilisation des services d'Érudit (y compris la reproduction) est assujettie à sa politique d'utilisation que vous pouvez consulter en ligne.

https://apropos.erudit.org/fr/usagers/politique-dutilisation/ 


\section{Positions et stratégies des éditeurs dans le champ éditorial québécois}

\section{Ignace Cau}

Après avoir identifié quatre axes possibles, l'auteur analyse le champ éditorial de différentes maisons québécoises de 1960 à 1977 et dégage l'image de ces éditeurs par rapport au marché et à leur stratégie éditoriale. L'évolution de l'édition devient lä̈que et idéologique au cours de cette période, mais est toujours faiblement structurée et partagée entre le livre "culturel" et le livre "pratique".

\section{The positions and strategies of Quebec publishers}

Having identified four possible points of reference, the author analyzes the activities of various publishing houses in Quebec from 1960 and 1977 relative to market and publishing strategy. Publishing became non-religious and ideological during this period, but was hardly structured and divided between "cultural" books and "practical" books.

\section{Las posiciones y estrategias de los editores en el mundo editorial quebecense}

Después de identificar cuatro orientaciones posibles, el autor analiza la situación de varias editoriales quebecenses del 1960 al 1977 y destaca la imagen de estos editores con relación al mercado y a su estrategia editorial. La evolución de la edición llega a ser laica e ideológica en este período, pero sigue siendo poco estructurada y vacila entre el libro "cultural" y el libro "práctico".

Ce texte, tiré d'une thèse de maîtrise en sociologie, porte sur le champ de l'édition au Québec au cours de la période 1960-1977. II va sans dire que plusieurs des éléments nécessaires à la compréhension et surtout à l'explication de notre objet d'étude sont absents de ces pages où nous tentons d'analyser les positions et les stratégies des éditeurs dans le champ éditorial québécois ${ }^{1}$.

Ce texte a été rédigé à partir des résultats d'une enquête par questionnaire effectuée au printemps 1978 auprès de 23 maisons d'édition. Le point de vue adopté ici pour situer les éditeurs interrogés dans

1. Les presses universitaires québécoises (PUM, PUL, PUQ) ne figurent pas dans ce texte. Elles ont fait l'objet d'une deuxième étude dans le cadre de notre recherche. le champ des positions (i.e. les maisons d'édition dans le champ éditorial) s'inspire des principes proposés par Pierre Bourdieu dans son article "La production de la croyance, contribution à une économie des biens symboliques"' 2. Pour définir l'espèce de capital qui est à la fois l'enjeu de la concurrence dans le champ et la condition de l'entrée dans cette compétition, Pierre Bourdieu caractérise les maisons d'édition à partir du cycle de vie de leur production. II y a les entreprises "commerciales", qui ont un cycle de production court fondé sur le souci de minimiser les risques financiers par un ajustement anticipé à la demande. Le cycle de production court est le produit d'un système de production dominé par la

2. Pierre Bourdieu, Actes de la recherche en sciences sociales, no 13 (février 1977), 3-44. 
recherche de rentabilité; ses ouvrages sont voués à un vieillissement rapide. Les entreprises "culturelles", elles, ont un cycle de production long fondé sur l'acceptation du risque inhérent aux investissements culturels qui prennent de la valeur avec le temps.

Nous avons donc caractérisé les différentes maisons d'édition selon la part qu'elles font aux investissements de risque à long terme et aux investissement sûrs à court terme. Pour construire le champ des positions et situer les éditeurs interrogés, nous avons particulièrement examiné les indicateurs suivants:

- la taille de l'entreprise et le volume de production (voir Tableau 1): les caractéristiques économiques de la maison définissent les limites à l'intérieur desauelles peuvent jouer les politiques d'édition; la structure financière commande toute politique éditoriale:

- les caractéristiques propres des éditeurs: leur affiliation prédominante au champ intellectuel et idéologique ou au champ économique; la raison par laquelle ils se trouvent dans le monde de l'édition; relation entre leurs discours et la pratique éditoriale;

- les caractéristiques de la production;

- l'âge des entreprises;

- le régime de propriété: particuliers, sociétés, associations, etc.; nous nous attarderons sur les maisons à affiliation idéologique ou confessionnelle.

Bref, à l'aide de leurs propriétés réelles (i.e. caractéristiques économiques, caractéristiques de la production et des éditeurs) et subjectives (i.e. représentations que les éditeurs se font de leur propre pratique), nous avons pu situer les maisons d'édition selon les quatre axes suivants: axe culturel, axe économique, axe culturel - idéologique, axe culturel - économique.

Le Tableau 2 présente les positions occupées par les éditeurs dans la structure du champ éditorial:

\section{Tableau 1}

\begin{tabular}{|c|c|c|c|}
\hline \multicolumn{4}{|c|}{$\begin{array}{l}\text { MAISONS D'ÉDITION QUÉBÉCOISES: } \\
\text { taille mesurée surtout au volume du chiffre d'affaires }\end{array}$} \\
\hline & $\begin{array}{l}\text { CLASSEMENT } \\
\text { SELON LA TAILLE }\end{array}$ & CHIFFRE D'AFFAIRES & $\begin{array}{l}\text { MOYENNE } \\
\text { PUBLICATION }\end{array}$ \\
\hline 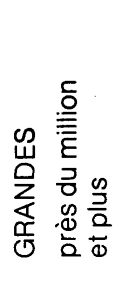 & $\begin{array}{l}\text { - Beauchemin } \\
\text { - Homme } \\
\text { - Fides } \\
\text { - Leméac } \\
\text { - Hurtubise HMH } \\
\text { - Jour } \\
\text { - Héritage } \\
\text { - La Presse } \\
\text { - Stanké }\end{array}$ & $\begin{array}{c}6-10 \text { millions } \\
5 \text { millions } \\
1 \text { million } \\
1 \text { million } \\
1 \text { million } \\
1600000 \\
1600000 \\
400000 \\
1 \text { million }\end{array}$ & $\begin{array}{r}30 \\
50 \\
55 \\
50 \\
30 \\
35 \\
50 \\
30 \\
\pm 40\end{array}$ \\
\hline 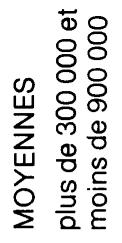 & $\begin{array}{l}\text { - Aurore } \\
\text { - Étincelle } \\
\text { - Naaman } \\
\text { - Quinze } \\
\text { - Bellarmin } \\
\text { - Paulines } \\
\text { - Québec/Amérique }\end{array}$ & $\begin{array}{l}400000 \\
500000 \\
350000 \\
500000 \\
300000 \\
300000 \\
342000\end{array}$ & $\begin{array}{c}30 \\
29 \\
30-40 \\
40-50 \\
15 \\
25 \\
15\end{array}$ \\
\hline 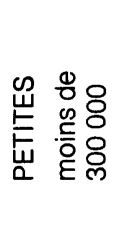 & $\begin{array}{l}\text { - CLF } \\
\text { - Hexagone } \\
\text { - Boréal-Express } \\
\text { - Parti-Pris } \\
\text { - Noroît } \\
\text { - Saint-Yves } \\
\text { - VLB }\end{array}$ & $\begin{array}{c}200000 \\
25-30000 \\
125 \cdot 150000 \\
150000 \\
10000 \\
20000 \\
125000\end{array}$ & $\begin{array}{r}20 \\
10 \\
10 \\
21 \\
10 \\
5 \\
\pm 10\end{array}$ \\
\hline
\end{tabular}


Tableau 2

\begin{tabular}{|c|c|c|c|}
\hline $\begin{array}{c}\text { AXE } \\
\text { CULTUREL }\end{array}$ & $\begin{array}{c}\text { AXE } \\
\text { CULTUREL- } \\
\text { IDÉOLOGIQUE }\end{array}$ & $\begin{array}{c}\text { AXE } \\
\text { CULTUREL- } \\
\text { ECONOMIQUE }\end{array}$ & $\begin{array}{c}\text { AXE } \\
\text { ÉCONOMIQUE }\end{array}$ \\
\hline $\begin{array}{l}\text { Hexagone } \\
\text { CLF } \\
\text { VLB } \\
\text { Noroît }\end{array}$ & $\begin{array}{l}\text { Parti-Pris } \\
\text { Boréal-Express } \\
\text { Fides } \\
\text { Bellarmin } \\
\text { Paulines }\end{array}$ & $\begin{array}{l}\text { Aurore } \\
\text { Leméac } \\
\text { HMH } \\
\text { Naaman } \\
\text { Quinze }\end{array}$ & $\begin{array}{l}\text { de l'Homme } \\
\text { du Jour } \\
\text { Stanké } \\
\text { La Presse } \\
\text { Héritage } \\
\text { Étincelle } \\
\text { Québec/Amérique } \\
\text { Beauchemin }\end{array}$ \\
\hline
\end{tabular}

\section{AXE CULTUREL ou la dénégation de l'économie}

\section{Image de l'éditeur "culturel"}

C'est la "dénégation de l'économie" qui est placée au coeur même du souschamp culturel. Une caractéristique de la relation de l'éditeur dit culturel à sa pratique, c'est qu'elle s'accompagne - beaucoup plus que celle des autres éditeurs d'un "refus du commercial". Tous les éditeurs culturels en effet se définissent par un refus du commercial. Tous affirment être dans le monde de l'édition "par amour de la littérature". Pour trois de nos informateurs, l'éditeur est le "possible" de la littérature, le lieu par lequel la littérature accède à l'existence: "l'éditeur donne la parole aux écrivains" (Hexagone), "il donne l'occasion à des forces créatrices latentes d'apparaître" (Noroît), "son rôle est d'apporter un prolongement à la création au niveau d'un objet qui s'appelle livre'" (VLB).

Les représentations de la fonction d'éditeur telles qu'annoncées par nos répondants ne sont pas de simples opinions qui ne se traduiraient pas dans la pratique du métier. Nous allons voir les stratégies mises de l'avant pour la réalisation de tels objectifs.

\section{Stratégies et rapport au marché}

Les maisons culturelles ont une pratique éditoriale bien définie, elles sont fidèles à leur image de marque ou à leur vocation première. Leur production - poésie, roman, essai, études littéraires et historiques - est à cycle de production long, fondé sur l'acceptation du risque inhérent aux investissements culturels qui prennent de la valeur avec le temps; cette production s'adresse à un public restreint (tirage faible ou peu élevé qui s'écoule lentement).

Les éditeurs culturels sont conscients d'être défavorisés dans le champ éditorial québécois qui est de plus en plus soumis à la rentabilité économique; par le fait même, la loi du marché est contraignante pour l'édition culturelle. Ils ont, par conséquent, organisé leurs maisons de sorte qu'ils puissent composer avec les contraintes économiques, sans faire de compromis inacceptables.

Les maisons culturelles peuvent composer avec les contraintes économiques et rester fidèles à leur vocation grâce à une structure éditoriale artisanale. "Nous autres, on n'a pas de frais généraux, pas de salaires à payer ... nous autres on n'est pas obligés de produire à tout prix pour faire vivre la maison et les gens qui travailient, ce qui nous évite des compromis de toutes sortes au niveau de la qualité de la production."

Un des compromis inacceptables serait, pour tous nos répondants, de faire du cloisonnement au niveau de la production (i.e. publier des livres de recettes pour pouvoir financer des romans). L'éditeur idéal n'est pas celui qui mélange le tout; il ne fait pas oeuvre de "rééquilibrage". "L'éditeur idéal ne publie pas des auteurs qui pensent faire oeuvre sérieuse à côté de n'importe qui ou de n'importe quoi; cela dévalorise ce que vous faites." En plus de disqualifier l'éditeur, le cloisonnement serait aussi un danger pour la santé financière de la maison. Un informateur nous dit: "je ne crois pas à l'édition polyvalente pour payer des oeuvres littéraires, les maisons 
qui l'ont fait sont tombées par terre (ex. Quinze et les Éditions du Jour)."

Par sa structure financière (relation dialectique entre le volume des affaires et la taille de l'entreprise), l'édition culturelle n'est pas obligée de rentabiliser le capital à tout prix. L'éditeur culturel refuse de grossir par le profit et pour le profit (dénégation de l'économie); les bénéfices, il les réinvestit toujours dans la production. L'éditeur culturel évite surtout "d'emballer la machine" par un nombre trop élevé de titres.

Favorisé par la taille de l'entreprise et le volume de la production, contrairement aux gros éditeurs, l'éditeur culturel prend lui-même connaissance des manuscrits reçus. C'est lui qui prend la décision de publier, après consultation de quelques amis de la maison qui sont le plus souvent ses auteurs. II ne commande pas de manuscrits; ceux-ci arrivent en grande partie d'auteurs qui écrivent déjà pour la maison. II est fréquent qu'un éditeur culturel accepte un manuscrit refusé par un al're éditeur: "ces manuscrits, nous dit-on, ont été refusés parce qu'ils étaient des oeuvres en travail. Pour des raisons de rentabilité, les autres éditeurs n'ont pas pris le temps de retravailler le manuscrit avec l'auteur". Donc, s'il ne suscite pas des manuscrits et qu'il se borne à les attendre (rôle passif), une fois en possession du manuscrit jugé valable, l'éditeur culturel le travaille plus que quiconque, au niveau du contenu et de la forme. Le livre pour les maisons culturelles est aussi un objet à toucher, elles le soignent d'une façon remarquable au niveau de la présentation graphique. Les éditions de l'Hexagone, par exemple, sont celles qui, au cours des années 1950, ont contribué le plus à changer le paysage graphique de l'édition québécoise; aujourd'hui, VLB éditeur semble s'imposer au niveau de l'originalité et de la présentation graphique du livre.

\section{Système capitaliste et marginalisation de la création}

Pour le système capitaliste, le livre est un objet de consommation quelconque; les appareils économiques de la distribution du livre poussent systématiquement le livre à rotation rapide (best-seller) en margina- lisant la création. L'édition littéraire demande une mise en marché appropriée, la littérature a besoin de centres de diffusion sélectifs, elle a besoin de rester sur les tablettes et non pas d'être traitée comme une nouveauté (best-seller) qui se démode vite, dont la vie est éphémère et qui doit par conséquent être remplacée rapidement. Pour toutes ces raisons, VLB éditeur, les éditions de l'Hexagone, le Noroît et d'autres éditeurs ont fondé les Messageries littéraires des éditeurs réunis.

Les maisons culturelles arrivent à se faire un nom, à devenir même un haut-lieu de la poésie francophone actuelle (ex. l'Hexagone), à se faire "un capital de consécration impliquant des pouvoirs de consacrer des personnes... donc de donner valeur et de tirer profit de cette opération" (P. Bourdieu), mais n'arrivent pas à recueillir pleinement les profits économiques de leur capital symbolique (culturel) parce que le système capitaliste de la distribution du livre limite la diffusion de la littérature (par exemple, un recueil de poésie d'un auteur important atteint le chiffre de 150 exemplaires après 8 mois de vente). Bien sûr, le marché restreint et le contrôle étranger de la distribution sont aussi des facteurs qui entrent en ligne de compte.

Bref, la loi du marché est contraignante pour les éditeurs culturels. Ils sont amenés par leur situation difficile à disqualifier les autres éditeurs, les "nouveaux marchands du temple", en prêchant pour les infidèles la conversion et le retour aux sources, i.e. la dénégation de l'économie.

Même si elles craignent les politiques gouvernementales en matière d'édition, ces quelques maisons d'édition ne refusent pas les subventions que les gouvernements fédéral et provincial mettent à leur disposition. Mais, de toute évidence, les politiques gouvernementales, axées sur le succès commercial, ne peuvent pas s'appliquer à ce type d'édition. 


\section{AXE ÉCONOMIQUE ou le livre comme source de profit immédiat}

\section{Image de l'éditeur "économique"}

La caractéristique principale de la relation de l'éditeur de l'axe économique à sa pratique, c'est qu'elle s'accompagne non pas d'un refus du commercial ou de la dénégation de l'économie, comme c'est le cas dans tous les autres axes ou souschamps éditoriaux, mais d'une préoccupation économique déclarée. La loi du champ économique, c'est la recherche de rentabilité; le livre est traité comme source de profit immédiat.

L'éditeur dit économique ne cache pas les principes qui animent sa pratique éditoriale. Pour nos répondants, "l'éditeur est un technicien du livre dont il doit assurer la rentabilité; il refuse un manuscrit quand il ne croit pas à sa rentabilité". L'édition est purement un "commerce"; si l'édition québécoise va mal, c'est parce que les éditeurs sont "des mauvais administrateurs". L'éditeur est "un agent commercial qui travaille entre le lecteur et l'écrivain pour faire en sorte que la littérature soit rentable". L'éditeur économique ne comprend pas le refus de certains éditeurs québécois de s'adapter aux lois commerciales; pour lui, chaque livre doit être rentable, et "ce n'est pas la tâche de l'éditeur de subventionner la littérature"'.

Aux éditeurs qui prêchent la dénégation de l'économie et qui cherchent à les disqualifier par l'anathème "marchands du temple", les éditeurs économiques répliquent d'" 'aller prendre des cours de marketing" et de "s'adapter aux lois commerciales", et leur conseillent aussi d'arrêter d'afficher des comportements d'assistés sociaux en voulant toujours rester attachés aux "mamelles de l'État". Les subventions ne permettent pas, selon l'éditeur économique, que s'opère une sélection naturelle parmi les maisons d'édition, parce qu'elles empêchent les meilleures de s'imposer.

Les luttes qui se déroulent dans le champ de l'édition québécoise engagent toujours le rapport à l'économie. Pour l'éditeur économique, affirmer que le livre ou la littérature doit être rentable et donc afficher une préoccupation économique dans la pratique éditoriale ne signifie nullement reconnaître que les livres soient dépourvus de valeur culturelle. II croit contribuer à l'enrichissement du patrimoine culturel québécois autant que l'éditeur culturel; il croit être présent à l'évolution intellectuelle du Québec même en publiant des livres de recettes (livres utilitaires) et en faisant de l'édition journalistique (i.e. livres-articles traitant de l'actualité sociale et politique). Selon les éditeurs de l'axe économique, la culture englobe toutes les manifestations de la vie. Ce n'est donc pas péjoratif pour eux de faire des livres utilitaires (livres qui répondent aux besoins quotidiens du public).

Le répondant de la maison la plus représentative du pôle économique (les Éditions de l'Homme) affirme publier des livres qui permettent aux gens d'aller plus loin, en donnant l'information la plus juste possible. "La production des Éditions de l'Homme colle avec l'évolution de'la société québécoise; on est à l'écoute du public, on dépend du public mais plusieurs livres ont aussi créé le goût du public et l'ont fait évoluer (ex.: I'Encyclopédie des Antiquités du Québec ou l'Encyclopédie de la Maison québécoise)."

\section{Structure financière et stratégies éditoriales}

Plus que dans tout autre axe éditorial, la structure financière et le type de production commandent la politique de la maison et le rapport au marché. Chez les maisons de grande taille, en particulier les Éditions de l'Homme et Beauchemin, l'investissement risqué à long terme est interdit.

Les maisons de l'axe économique se classent toutes, sauf Étincelle et Québec/ Amérique, parmi les maisons de grande taille avec une structure éditoriale professionnelle. Beauchemin et les Éditions de l'Homme sont de véritables industries éditoriales parfaitement intégrées aux rouages de l'économie capitaliste. Chez 
ces deux maisons, on peut parler d'industrialisation extensive et intensive ${ }^{3}$.

À cause du poids des frais généraux élevés, les grosses maisons ne peuvent prendre le temps qu'exige la conversion en capital culturel. La production de l'axe économique est presqu'exclusivement à cycle de production court (tirages élevés et écoulement de stock rapide) fondé sur le souci de minimiser les risques par un ajustement anticipé à la demande (on com. mande beaucoup de manuscrits à partir des besoins existants).

Les Éditions de l'Homme sont la maison la plus représentative de l'axe économique: $80 \%$ de sa production est à proprement parler du livre pratique sur la vie quotidienne, livre à rotation rapide. Le livre populaire a fait son entrée sur le marché de l'édition québécoise exactement avec la fondation des Éditions de l'Homme en 1957. Mais jusqu'à 1968, date d'affiliation au groupe Sogides, la production des Éditions de l'Homme était diversifiée. Le livre culturel ou littéraire faisait bon ménage avec le livre pratique. Ce difficile équilibre n'a plus été possible depuis que Sogides est devenu l'acquéreur des Éditions de l'Homme. En accroissant la taille de l'entreprise, donc les frais généraux et les volumes de production, les Éditions de l'Homme ont vite compris que les investissements risqués à long terme leur étaient dorénavant interdits. Elles ont accepté la dialectique du profit en laissant de côté le livre culturel (la littérature) pour se spécialiser dans le livre de vulgarisation. Pour des raisons de rentabilité, toute publication des Éditions de l'Homme doit avoir un tirage supérieur à 8000 exemplaires et doit s'écouler dans l'espace de douze à quinze mois.

Les Éditions du Jour, la maison d'édition la plus représentative de la renaissance

3. Aux éditions Beauchemin et de l'Homme, l'industrialisation extensive est de type vertical et horizontal. Vertical: elle s'accomplit a) en aval, contrôlant les produits nécessaires à la fabrication et s'attachant le maximum d'auteurs spécialisés (dans l'enseignement et dans le livre de vulgarisation); b) en amont, dans la fabrication (imprimerie) et dans la commercialisation (librairies et messageries). Horizontal: c'est le cas des Éditions de l'Homme qui contrôlent aussi deux autres maisons, l'Actuelle et les Presses libres. L'industrialisation intensive consiste dans la rationalisation des méthodes de gestion. intellectuelle du Québec des années 1960, fait, elle aussi, des ouvrages de vulgarisation sur des sujets comme la psychologie, la parapsychologie, la santé, la coopération et des livres pratiques, en visant un public très large. Des difficultés financières ont obligé Jacques Hébert, en 1973, à vendre aux Caisses d'économie du Québec qui, elles, ont donné une nouvelle orientation à la maison d'édition. Pour Jacques Hébert, publier, c'est d'abord servir la liberté de créer, de penser et de dire; les livres pratiques servent de "locomotives" et fournissent les moyens financiers pour s'imposer sur le plan littéraire. Les Éditions du Jour ont imposé les noms de Marie-Claire Blais, J. Ferron, J. Benoit, Victor-Lévy Beaulieu, Roch Carrier et Gatien Lapointe. En treize ans (1961-1973), les Éditions du Jour ont publié 600 titres. Un tel volume de production interdisait aux responsables de la maison d'investir dans des ouvrages à cycle de production long. Jacques Hébert a été incapable d'entretenir une relation dialectique entre le volume d'affaires et la taille de l'entreprise par des stratégies qui se fondaient sur les deux structures: culturelle et économique. Il est facile de saisir les contradictions qui résultent des incompatibilités entre les deux économies: le type de gestion ou d'organisation qui convient pour produire, diffuser et faire valoir une catégorie de livres est parfaitement inadapté pour l'autre. Ceci explique en partie la chute de la maison de Jacques Hébert.

La maison Beauchemin, l'éditeur des premiers romans d'Anne Hébert, qui a encore dans son catalogue sept romans de Gabrielle Roy, investit toujours plus dans le manuel scolaire, livre utilitaire qui reste à cheval entre le culturel et l'économique. $80 \%$ des investissements de Beauchemin vont au manuel scolaire, $20 \%$ est consacré à la littérature: littérature au sens strict et littérature populaire. La production de manuels scolaires, une catégorie de livres qui nécessite de grands investissements et qui vieillit vite dans un système scolaire mobile, et la taille de l'entreprise (environ 10 millions de dollars de chiffre d'affaires) avec un poids de frais généraux très lourd lui interdisent de gros investissements à long terme; il doit faire tourner rapidement un capital essentiellement économique.

La production des Éditions Stanké est 
axée sur l'actualité et l'événement; elles visent à "distraire, documenter, informer et susciter la réflexion sur tous les plus importants points de notre temps". C'est une production à rotation rapide et à tirages élevés. Produire des "livres-événements" c'est une tâche très coûteuse; il faut donc que les capitaux rentrent vite pour la bonne marche de la maison. Si les Éditions Stanké publient de temps à autre des ouvrages de littérature, ouvrages à cycle de production long, c'est, au dire de son répondant, "pour l'image de marque de la maison", parce qu'un grand éditeur doit publier de la littérature. D'autre part, les Éditions Stanké, en littérature, éditent la "crème" ou des auteurs connus; elles ne lancent pas de jeunes auteurs (inconnus) pour ne pas "fermer rapidement". En d'autres mots, les Editions Stanké publient de la littérature à condition qu'elle se vende. Un pourcentage trop élevé d'ouvrages littéraires "compromet l'avenir de la maison", laquelle est axée principalement sur le livre à rotation rapide.

La production des Éditions La Presse est à l'image du journal du même nom: elle est de qualité, mais axée sur l'actualité. Presque $70 \%$ de la production a un caractère commercial: les tirages sont élevés et l'écoulement de stock rapide. Le restant. soit $30 \%$, est consacré à la littérature (romans, essais, anthologies).

La maison Héritage publie des livres d'intérêt général pour répondre aux besoins d'un public de masse (biographie, romanmystère, télé-succès, astrologie, littérature enfantine), une production qui est, dans son ensemble, à rotation rapide.

Enfin, L'Étincelle et Québec/Amérique, maisons que l'on sera peut-être surpris de voir classées dans l'axe économique, auraient pu être à la limite situées dans l'axe culturel-économique, mais il nous semble que dans le difficile équilibre entre l'approche culturelle et commerciale, c'est l'approche économique qui l'emporte.

Les Éditions Québec/Amérique offrent au public des textes surtout axés sur l'actualité politique québécoise, canadienne et internationale, "des textes qui suscitent la discussion et la réflexion". Bref, leur production, bien que de qualité, est presqu'ex- clusivement à cycle de production court: tirages élevés et écoulement de stock rapide. Beaucoup de titres doivent s'écouler dans l'espace de trois mois.

La production de l'Étincelle, très variée, touche des sujets de l'actualité et de la vie quotidienne. Les trois quarts de la production sont des traductions, lesquelles sont en grande partie des best-sellers, donc tirages élevés et cycle de production court.

\section{Rapport au marché}

Pour les maisons situées dans l'axe économique, qui traitent le livre comme une source de profit immédiat, la situation financière n'est pas difficile, elle est même prospère. Les appareils économiques de la distribution privilégient ce genre de production à rotation rapide. La Presse, Québec/Amérique et Stanké sont distribuées par les Nouvelles messageries internationales du livre (N.M.I.L.), la puissante maison de distribution contrôlée par Hachette. Les Éditions de L'Homme et les Éditions du Jour sont distribuées par l'Agence de distribution populaire (A.D.P.), le seul distributeur québécois qui soit en mesure de concurrencer les distributeurs étrangers. Les Éditions Étincelle sont distribuées par Dimedia, maison de distribution étrangère; Beauchemin et Héritage sont distribuées par leurs propres réseaux.

\section{AXE CULTUREL-IDÉOLOGIQUE ou l'édition comme vocation}

Les maisons de l'axe culturel-idéologique se caractérisent par la place plus grande faite à la réflexion morale et politique. L'éditeur dit culturel-idéologique se définit lui aussi par un refus du commercial, mais les définitions qu'il nous donne de sa pratique entretiennent un lien nécessaire soit avec le champ intellectuel soit avec le champ idéologique (politico-religieux).

\section{Maisons religieuses: Fides, Bellarmin, Paulines}

Les maisons Fides, Bellarmin et Paulines sont l'oeuvre de trois communautés religieuses qui sont responsables de leur politique: respectivement la Congrégation 
Sainte-Croix, la Compagnie de Jésus et la Société Saint-Paul.

Image de l'éditeur "religieux"

Les définitions que les éditeurs religieux donnent de leur pratique sont, pour l'essentiel, identiques. Selon nos répondants, "l'éditeur appartient au domaine de la pensée et de l'apostolat'. Le livre, tout en étant un instrument culturel, est aussi un instrument "d'évangélisation". Ces trois maisons se sont données comme finalité de travailler pour "réaliser l'homme intégral" (i.e. humanisme intégral selon la vision de Maritain: harmoniser les valeurs chrétiennes et les valeurs de la civilisation). Elles ne se sont jamais confinées à des sujets religieux. "L'éditeur voit les besoins de son époque et cherche à y répondre en acceptant et suscitant des manuscrits." L'éditeur doit "promouvoir quelque chose de bien et de beau, il est l'homme de la nouveauté"; le "bien", pour ce groupe d'éditeurs, est tout ce qui concourt à promouvoir les valeurs humaines et chrétiennes; "nouveau" désigne le rôle actif que doit jouer l'éditeur. II n'attend pas que les manuscrits lui arrivent (rôle passif); il en commande aussi, soit pour répondre à un besoin existant, soit pour créer des débats et de nouvelles orientations de pensée.

\section{Rapport au marché et stratégies}

La maison Fides se classe parmi les grandes entreprises d'édition au Québec; sa structure éditoriale est professionnelle. Les maisons Bellarmin et Paulines, qui ont une structure éditoriale semi-professionnelle, se classent parmi les maisons de taille moyenne. La forme juridique et l'âge de l'entreprise sont les deux variables essentielles pour l'analyse de ces trois maisons.

Les maisons Fides, Bellarmin et Paulines sont des corporations, entreprises constituées à but non lucratif; elles ont été fondées avant les années 1960, dans une période où le champ éditorial québécois était totalement soumis à la légitimité idéologique ou religieuse (avant 1960, on assiste à une grande production de vulgarisation religieuse, l'édition scolaire et le commerce du livre sont entre les mains de communautés religieuses, un très grand nombre de librairies relève des procures). Les trois maisons religieuses pour lesquelles le rapport au marché n'était pas contraignant jusqu'à la décennie 1960 ont pu pratiquer pour la même période une industrialisation extensive de type vertical (i.e. imprimeries propres, librairies, revues et autres activités parallèles).

Au cours des années 1960, le champ éditorial québécois a été soumis à de nouvelles instances de légitimation (i.e. écrivains, journalistes, universitaires, vont supplanter l'ancienne élite cléricale dans la tâche de fournir des symboles et des valeurs à la pensée collective); les maisons religieuses ont dû se recycler au niveau des catégories de production et au niveau de la structure éditoriale. La maison Fides abandonne à partir de 1970 ses activités en aval et en amont; elle s'est débarrassée de dix librairies (elle n'en garde qu'une), des revues et de son imprimerie; par contre, elle contrôle encore la distribution de ses livres par la vente en gros et au détail. Bellarmin et Paulines contrôlent elles aussi la distribution de leurs livres par la vente en gros et au détail, respectivement par une et deux librairies. Bellarmin et Paulines publient aussi des revues. Bellarmin en gère trois, dont Relations, Paulines publie Vidéo-Presse, une revue pour les jeunes.

En ce qui concerne l'évolution de catégories de production, on remarque que le changement s'est opéré au début des années 1960 avec la sécularisation de la société québécoise. Après avoir toujours privilégié la production du livre religieux, Fides, Bellarmin et Paulines ont réduit progressivement le volume de cette production; dans la décennie 1960, elles ont consacré à la production religieuse seulement la moitié de leurs investissements, pour les rendre encore moins importants au cours des dernières années. Fides privilégie, actuellement, l'édition littéraire et historique (i.e. $50 \%$ de son investissement). Le livre religieux, quant à lui, va chercher à peu près $25 \%$ de l'investissement; le restant est octroyé à l'édition de littérature de loisir et de jeunesse.

Les Éditions Paulines consacrent une grande partie de leur investissement à la littérature enfantine. Pour l'année financière 1977, l'investissement était ainsi réparti: 
$51,6 \%$ pour la littérature enfantine, 34,4\% pour les livres religieux et $14 \%$ en sciences humaines.

Enfin, Bellarmin consacre à l'édition religieuse presque la moitié de son investissement; le restant est octroyé à l'édition savante (philosophie, sociologie, éducation) et à quelques titres s'adressant à un public plus large.

Bref, il nous semble que les maisons religieuses produisent dans l'ensemble des livres à cycle de production long (i.e tirages pas très élevés et écoulements de stock relativement longs). Elles peuvent réinvestir dans une édition à cycle de production long moyennant trois facteurs: leurs fonds d'édition, les subventions gouvernementales et le statut juridique de l'entreprise. Ces facteurs se révèlent déterminants particulièrement pour les Éditions Fides à qui les caractéristiques structurales interdisent une production tournée vers le cycle de production long. Fides est parmi les rares maisons québécoises qui tirent un profit économique du capital culturel accumulé. Née pendant la guerre, comme beaucoup d'autres, Fides sera l'une des rares maisons à survivre grâce à son fonds d'édition. A la fin de la guerre, elle a accumulé moins de liquidité que ses concurrentes, mais elle se trouve riche de près de 200 titres originaux. La collection Nénuphar par exemple, lancée en 1944, vit toujours.

\section{Boréal-Express et Parti-Pris}

Image de marque

Boréal-Express et Parti-Pris privilégient la réflexion politique. Boréal-Express a, comme but principal, "de contribuer au réveil de la conscience nationale québécoise". Cette maison cherche à développer la conscience nationale en cultivant la conscience historique par la publication du livre d'histoire (histoire politique, urbaine et sociale). Son but a toujours été de fournir "des matériaux pour une réflexion sérieuse, les diffuser le plus largement possible mais sans jamais imposer une école d'historiens pas plus qu'une action politique".

Parti-Pris, pour sa part, se définit comme maison "de combat". Elle incarne la première analyse radicale de la réalité québécoise. Cette maison entend être plus que jamais "le point d'ébullition de la littérature, des idées, le miroir de l'émergence d'un peuple, le nôtre. . être au service de la civilisation québécoise".

Tournée vers un cycle de production long, la production de Boréal-Express et Parti-Pris a su provoquer, au cours des années 1960, une parole collective qui a contribué à donner aux Québécois une meilleure perception d'eux-mêmes.

\section{Rapport au marché}

Boréal-Express et Parti-Pris se classent parmi les maisons de petite taille avec lesquelles elles partagent les caractéristiques structurales (i.e. structures artisanales) et les problèmes de mise en marché. Pour Boréal-Express, le rapport au marché est plus facile que pour Parti-Pris; en effet, sa production donne des fruits économiques relativement rapidement parce que l'écoulement des stocks pour les ouvrages d'histoire est généralement moins long. Parti-Pris doit faire face aux mêmes problèmes que l'édition de l'axe culturel, sa production étant à $54,4 \%$ littéraire.

Enfin Boréal-Express et Parti-Pris ont une structure éditoriale harmonisée à leur type de production. Les deux maisons évitent de produire un nombre de livres supérieur à leurs possibilités. Leur prudence économique consiste à grossir avec leurs propres moyens.

\section{AXE CULTUREL-ÉCONOMIQUE ou l'édition comme reflet de "cultures québécoises"}

\section{Image de l'éditeur "culturel-économique"}

L'éditeur dit culturel-économique se définit lui aussi par le refus du commercial. "Le rôle de l'éditeur, nous dit un informateur, ce n'est pas celui de faire de l'argent; l'éditeur est un stimulateur public, il est un communicateur d'idées, il a un rôle d'animateur social; il doit faciliter et faire naître les débats, cerner les débats là où ils séjournent; il doit donner la parole à tout le 
monde". Chacun à leur façon, les éditeurs de l'axe culturel-économique cherchent à disqualifier les concurrents dans leur rapport à l'économie. Un répondant affirme par exemple: "depuis 22 ans, nous avons toujours gardé la ferveur nécessaire pour oeuvrer sans cesse dans un domaine où nous voyons les artisans se décourager et les promoteurs faire face à des politiques éditoriales plus rentables à cause des impératifs financiers plutôt que de conserver la volonté de publier romans, poésies, littérature québécoise en général".

Au niveau de l'image de marque, presque toutes les maisons se définissent par rapport au fait québécois. Elles cherchent à "refléter l'expérience québécoise" (Aurore), "à être un reflet des cultures québécoises" (Quinze), à "être québécois à $100 \%$ ou à être une maison spécialisée dans l'édition d'auteurs québécois" (Leméac).

En d'autres mots, la quasi-totalité des maisons publie des ouvrages touchant toutes les dimensions de la vie sociale québécoise: littérature, essais historiques, politiques et économiques, guides pratiques, ouvrages portant sur les loisirs et les sports, etc.

\section{Stratégies et rapport au marché}

La structure financière et la taille de l'entreprise des maisons du sous-champ culturel-économique varient d'une maison à l'autre. Les trois maisons de fondation récente, à savoir Naaman, Quinze et Aurore, fondées au cours des années 1970, ont une structure financière à mi-chemin entre le professionnel et l'artisanal et elles sont de taille moyenne. Les Éditions de l'Aurore et Quinze traversent une situation économique difficile; elles ont été plusieurs fois au bord de la faillite.

Pour HMH et Leméac, deux maisons plus anciennes et de plus grande taille, avec une structure éditoriale professionnelle, la situation économique est bonne. $\mathrm{HMH}$ et Leméac ont joué un grand rôle dans l'évolution culturelle québécoise. Ces deux maisons ont donné la parole à des écrivains prestigieux. Leurs animateurs sont d'ailleurs conscients de s'être fait un nom, capable de consacrer des auteurs et de tirer un profit économique du capital culturel accumulé. "Nous sommes conscients, nous dit le répondant de la maison Leméac, que nous pourrions céder à la tentation de nous adonner, grâce à la place que nous avons acquise, à la publication de livres faciles." Mais au nom de ces auteurs et du public, cet éditeur entend poursuivre la vocation première de sa maison. Cependant, il semble bien que cette maison ait déjà cédé, à cause des contraintes économiques, à la tentation de publier des livres faciles; par livres faciles, nous entendons ici les ouvrages qui s'adressent à un public plus large; citons les collections "mon pays et mes chansons", "recettes typiques", "éducation physique et loisirs", "dossiers", et enfin le fond pédagogique, une production chevauchant le culturel et l'économique.

La maison $\mathrm{HMH}$ opère dans deux grands domaines d'activité: l'édition littéraire et l'édition scolaire qui représente les deux tiers de la production globale. L'édition littéraire vit grâce aux livres scolaires, aux subventions gouvernementales et enfin à quelques livres pratiques.

À l'origine des maisons l'Aurore et Quinze, il y avait un besoin de littérature. La vocation principale de ces deux maisons était "la publication d'oeuvres romanesques", de publier le plus grand nombre de livres culturels à des "prix compétitifs" sans faire "la piasse facile" avec l'édition "rapide", qu'on nomme "pratique".

Dans un laps de temps très court, ces deux maisons ont permis à un grand nombre d'auteurs d'être publiés et distribués, mais elles n'ont pas été capables d'établir une relation dialectique entre le volume des affaires et la taille de l'entreprise et ont failli fermer leurs portes.

Les Éditions Quinze (qui ont publié des titres de G. Bessette, J. Godbout, M.-C. Blais, Y. Thériault, A. Major, G. Archambault, etc.) ont maintenant réduit le nombre de publications et diversifié leur production "pour établir un équilibre entre les livres destinés à un public restreint, qui s'écoulent lentement sur le marché, et ceux qui touchent l'actualité et qui s'adressent à une vaste clientèle".

La nouvelle stratégie adoptée par 
l'Aurore consiste à éviter d'entrer en concurrence avec d'autres éditeurs; pour cette raison, la maison a arrêté la production d'oeuvres proprement littéraires. Elle entend participer aux mouvements littéraires par d'autres aspects. 'L'homme littéraire n'est pas uniquement le romancier ou le poète; nous croyons fermement qu'il est possible de mettre autant de "littérature", autant d'imagination et de plaisir dans un manuel sur l'art de planter les clous que dans un roman." L'Aurore tente de légitimer les livres plus "prosaïques" au nom de I" "imagination pratique" et de l'"esprit de création pratique". D'après son répondant, l'édition québécoise ne peut se satisfaire d'une image "littéraire" si elle veut survivre; elle doit s'internationaliser - selon un point de vue québécois - et créer de nouveaux domaines d'activités pour ne pas entrer en concurrence avec les autres éditeurs. En somme, les "livres pratiques" servent en quelque sorte à la bonne marche de cette maison, pour laquelle les gros tirages ne sont pas le but premier.

La production des Éditions Naaman est surtout littéraire. La vocation de cette maison se veut internationale: "faire connaître la littérature de langue française hors de la France". Sa production, axée sur le culturel, s'alimente comme celle de tous les autres éditeurs du sous-champ culturel économique à des catégories plus rentables (i.e. les collections "livres pour tous", "lectures brèves", anthologies scolaires).

\section{Conclusion}

Pour rendre compte de la structuration actuelle du champ éditorial québécois (francophone), il faudrait prendre en considération divers facteurs (explicatifs). Par exemple, le seul fait que jusqu'à tout récemment le clergé ait eu le monopole du système d'enseignement et ait contrôlé diverses institutions culturelles explique la présence de grandes maisons religieuses. Par ailleurs, l' "explosion scolaire"', l'élévation du taux de scolarisation, le développement du système d'enseignement collégial et universitaire et l'apparition de nouveaux secteurs de production culturelle (télévision, cinéma, radio, etc.) ont favorisé la constitution d'un milieu intellectuel québécois plus dynamique et autonome, d'où l'émergence, au milieu des années 1960, de nombreuses maisons culturelles.

Toutefois, dans son ensemble, le champ éditorial québécois demeure fortement concurrencé, pour ne pas dire dépendant de l'édition étrangère (en particulier française); en dépit des supports (subventions, etc.) offerts par les gouvernements fédéral et provincial, il est, sauf quelques exceptions, fragile. De plus, le monde de l'édition québécoise apparaît encore faiblement structuré, c'est-à-dire qu'il n'y a pas une forte différenciation entre les diverses maisons d'édition: sauf pour le livre scolaire, il n'existe pas de véritable spécialisation, chacune des maisons publiant "un peu de tout".

Enfin, le secteur proprement culturel est lui-même restreint, fragile et peu différencié: il n'y a pas, comme en France. de grandes maisons d'édition culturelles. Les quelques mesures politiques, indispensables à la survie de la plupart des maisons d'édition, n'ont pas encore réussi à "corriger" la situation, d'autant moins que, le plus souvent, elles ne sont pas adaptées à la diversité du monde de l'édition québécoise. 